\title{
Investigation of Gas Phase Ion Structure for Proline-Containing $b_{2}$ Ion
}

\author{
Lori L. Smith,* Kristin A. Herrmann and Vicki H. Wysocki \\ Department of Chemistry, University of Arizona, Tucson, Arizona, USA
}

Unusual fragmentation was observed for doubly charged VPDPR in which cleavage Cterminal to proline and $\mathrm{N}$-terminal to aspartic acid yielded $\mathrm{b}_{2}\left(+\mathrm{a}_{2}\right) / \mathrm{y}_{3}$ complementary ions. This unique fragmentation is contradictory to trends previously established by statistical analysis of peptide tandem mass (MS/MS) spectra [1-4]. Substitution of alanine for aspartic acid (i.e., VPAPR) did not change the fragmentation, indicating the cleavage was not directed by aspartic acid. Fragmentation patterns for VPAPR and V(NmA)APR (NmA = N-methyl alanine) were compared to determine whether conformational constraints from proline's cyclic side-chain contribute to $b_{2}$ ion formation. While both peptide sequences fragmented to yield $b_{2} / y_{3}$ ions, only VPAPR produced $a_{2}$ ions, suggesting the VP $b_{2}$ ion is structurally different from the $\mathrm{V}(\mathrm{NmA}) b_{2}$ ion. Instead, the $\mathrm{V}(\mathrm{NmA}) b_{2}$ ion was accompanied by an ion corresponding to formal loss of 71 . The suspected structural differences were confirmed by isolation and fragmentation of the respective $\mathrm{b}_{2}$ ions (i.e., $\mathrm{MS}^{3}$ spectra). Evidence supporting a diketopiperazine structure for the VP $b_{2}$ ion is reported. Fragmentation patterns for the VP $b_{2}$ ion and a synthetic VP diketopiperazine showed great similarity. N-terminal acetylation of VPAPR prevented the formation of the VP $b_{2}$ ion, presumably by blocking nucleophilic attack by the $\mathrm{N}$-terminal amine on the carbonyl oxygen of the protonation site. Acetylation of the $\mathrm{N}$-terminus for $\mathrm{V}(\mathrm{NmA}) \mathrm{APR}$ did not prevent the formation of the $\mathrm{V}(\mathrm{NmA}) \mathrm{b}_{2}$ ion, indicating the $\mathrm{V}(\mathrm{NmA}) \mathrm{b}_{2}$ ion has a structure, presumably that of an oxazolone, which requires no attack by the N-terminus for formation. Finally, high-resolution, accurate mass measurements determined that the $\mathrm{V}(\mathrm{NmA})\left(\mathrm{b}_{2}-71\right)$ ion results from losing a portion of valine from oxazolone $\mathrm{V}(\mathrm{NmA}) b_{2}$ ion, rather than cross-ring cleavage of the alternate diketopiperazine. (J Am Soc Mass Spectrom 2006, 17, 20-28) (c) 2005 American Society for Mass Spectrometry

$\mathrm{M}$ ass spectrometry is playing a key role, in conjunction with other analytical tools (e.g., electrophoretic and multidimensional separations and sequence database searching algorithms), to elucidate gene function from protein expression profiles [5-9]. Peptide sequence information is obtained by using tandem mass spectrometry (MS/MS) and lowenergy collision induced dissociation (CID) to identify individual proteins. Useful algorithms like SEQUEST [10] and Mascot [11] have been developed to identify proteins by matching raw MS/MS CID spectra with peptide sequences from protein or translated nucleotide sequence databases. The predictive model of peptide fragmentation used by most current automated peptide sequencing algorithms anticipates cleavage of all amide bonds along the peptide backbone, presumably result-

Published online December 9, 2005

Address reprint requests to Dr. V. H. Wysocki, Department of Chemistry, University of Arizona, 1306 E. University Blvd., Tucson, AZ 85721, USA. E-mail: vwysocki@email.arizona.edu

* Names are necessary to report factually on available data; however, the USDA does not guarantee or warrant the standard of the product, and the use of the name by the USDA implies no approval of the product to the exclusion of others that may also be suitable.

* Also at USDA-ARS, Forage Animal Production Research Unit, Lexington, Kentucky, USA ing in the formation of $b_{n}$ and $y_{n}$ ions, using Roepstorff / Biemann nomenclature $[12,13]$. Other ion types are also formed that can be useful in automated peptide sequencing, including $a_{n}$ ions, immonium ions, internal sequence ions, and neutral losses of ammonia and water from these ions. Differences between the $\mathrm{m} / \mathrm{z}$ values of adjacent fragment ions of the same series can be correlated to the mass of an amino acid residue. Thus, a complete series of either of these ion types allows the sequencing of the entire fragmented peptide. Predicting spectral content using this model for peptide fragmentation has been proven successful on a fundamental level where automated protein identification can be achieved for many samples using database searching algorithms [14-20]. However, this simple model of peptide fragmentation occasionally fails to correctly predict the appearance of peptide MS/MS spectra, resulting in poor protein matches using database searching algorithms [21]. Attempts to understand the limitations of these algorithms and improve the fragmentation model are being made. Some recent strategies involve statistical analysis of fragmentation patterns of peptide MS/MS spectra [1-4, 22] and studying the chemistry involved in peptide fragmentation mechanisms [23-32] to more intelligently predict 


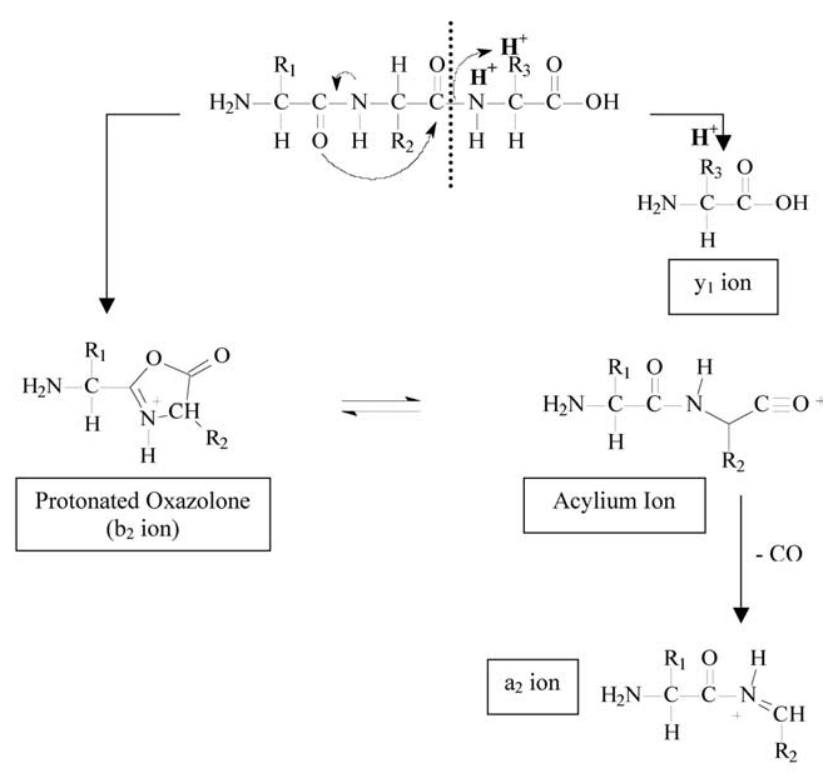

Scheme 1. Mechanism of peptide fragmentation to yield a protonated oxazolone $b_{2}$ ion and the corresponding $a_{2}$ ion from $a$ doubly protonated precursor ion.

MS/MS spectral appearance. Using both of these approaches, residue specific enhanced cleavages have been identified and investigated for peptides containing either proline or aspartic acid residues [1, 22, 26, 33-35]. Peptides containing either of these residues often produce incomplete ion series in the spectra, preventing sequencing of the entire peptide. Instead, preferential cleavage of the peptide bond occurs immediately $\mathrm{N}$ terminal to proline and C-terminal to aspartic acid residues, particularly when the number of basic residues (e.g., arginine, lysine, and histidine) in the sequence is greater than the number of ionizing protons, producing a few dominant fragment peaks in the spectra. Contrary to these residue specific trends, fragmentation of a pentapeptide (VPDPR in single letter abbreviations for amino acid residues) with proline and aspartic acid in the second and third residue positions, respectively, results in a MS/MS spectrum showing cleavage at the second peptide bond, C-terminal to proline and $\mathrm{N}$-terminal to aspartic acid. While formation of $a b_{2} / y_{3}$ ion pair from a pentapeptide is not unusual, cleavage $\mathrm{C}$-terminal to a proline residue is interesting. Based on current knowledge of peptide fragmentation trends for larger peptides ( $>5$ residues) where proline is located at an internal sequence position other than the first or second residue positions, one might expect cleavage $\mathrm{N}$-terminal to proline yielding formation of abundant $\mathrm{y}_{4}$ and $\mathrm{y}_{2}$ ions for VPDPR with little other sequencing information present in the spectrum. Further, $y_{n}$ ions are expected rather than $b_{n}$ ions because arginine's basic side-chain would encourage charge retention on the C-terminal portion of the peptide fragment. However, the observation that cleavage Cterminal to proline can and does occur when proline is the second residue in the peptide sequence has been noted on a larger scale in the statistical analysis of 5500 unique peptide MS/MS spectra as previously reported by Kapp et al. [2]. The primary objective of this research focuses on understanding gas-phase ion structure when proline is the C-terminal residue of $a b_{2}$ ion and the relative stabilities of such structures following formation.

The most common $b_{n}$ ion structure (where $n \geq 2$ ) is that of a protonated oxazolone [24, 36-40]. The formation of this ion type from a doubly protonated precursor ion proceeds through a mechanistic pathway involving charge-directed peptide fragmentation as illustrated in Scheme $\mathbf{1}$ [36, 37, 41-43]. Scheme $\mathbf{1}$ is shown with the protonation site on the nitrogen in the peptide bond for simplicity, yet protonation of the carbonyl oxygen may actually be involved. The lone pair of electrons, present in the carbonyl oxygen of the adjacent carbonyl $\mathrm{N}$ terminal to the site of protonation, attacks the electropositive carbonyl carbon at the protonation site. This initiates dissociation of the protonated peptide bond and rearrangement results in the formation of a cyclic protonated oxazolone ( $\mathrm{b}_{\mathrm{n}}$ ion) and the singly charged remaining portion of the peptide $\left(\mathrm{y}_{\mathrm{n}}\right.$ ion). Protonated oxazolone structures may undergo ring opening to form an acylium $b_{n}$ ion structure. It is from this acylium ion that $b_{n}$ ions may lose carbon monoxide to form corresponding $\mathrm{a}_{\mathrm{n}}$ ions. In fact, it has been reported [44] that the presence of $a b_{n} / a_{n}$ ion pair is often indicative of a protonated oxazolone structure for the corresponding $b_{n}$ ion.

The free amine moiety located on the $\mathrm{N}$-terminus of the peptide may initiate the formation of $b_{2}$ ions possessing a diketopiperazine structure [21, 40, 45] rather than the more common protonated oxazolone structure of larger $b_{n}$ fragments. The mechanism of diketopiperazine formation is shown in Scheme $\mathbf{2}$ and is essentially the same as that of protonated oxazolone formation. The main difference is that the lone pair of electrons on the N-terminal amino-nitrogen, rather than the electrons on the adjacent carbonyl oxygen, is the initiator of attack on the carbonyl carbon at the protonation site. The final $b_{2}$ ion structure is that of a protonated,

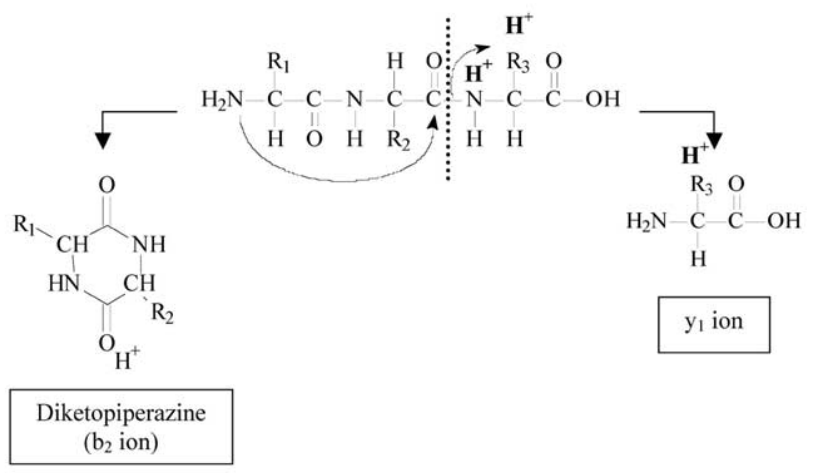

Scheme 2. Mechanism of formation of diketopiperazine $b_{2}$ ion and $a y_{1}$ ion from a doubly protonated precursor ion. 

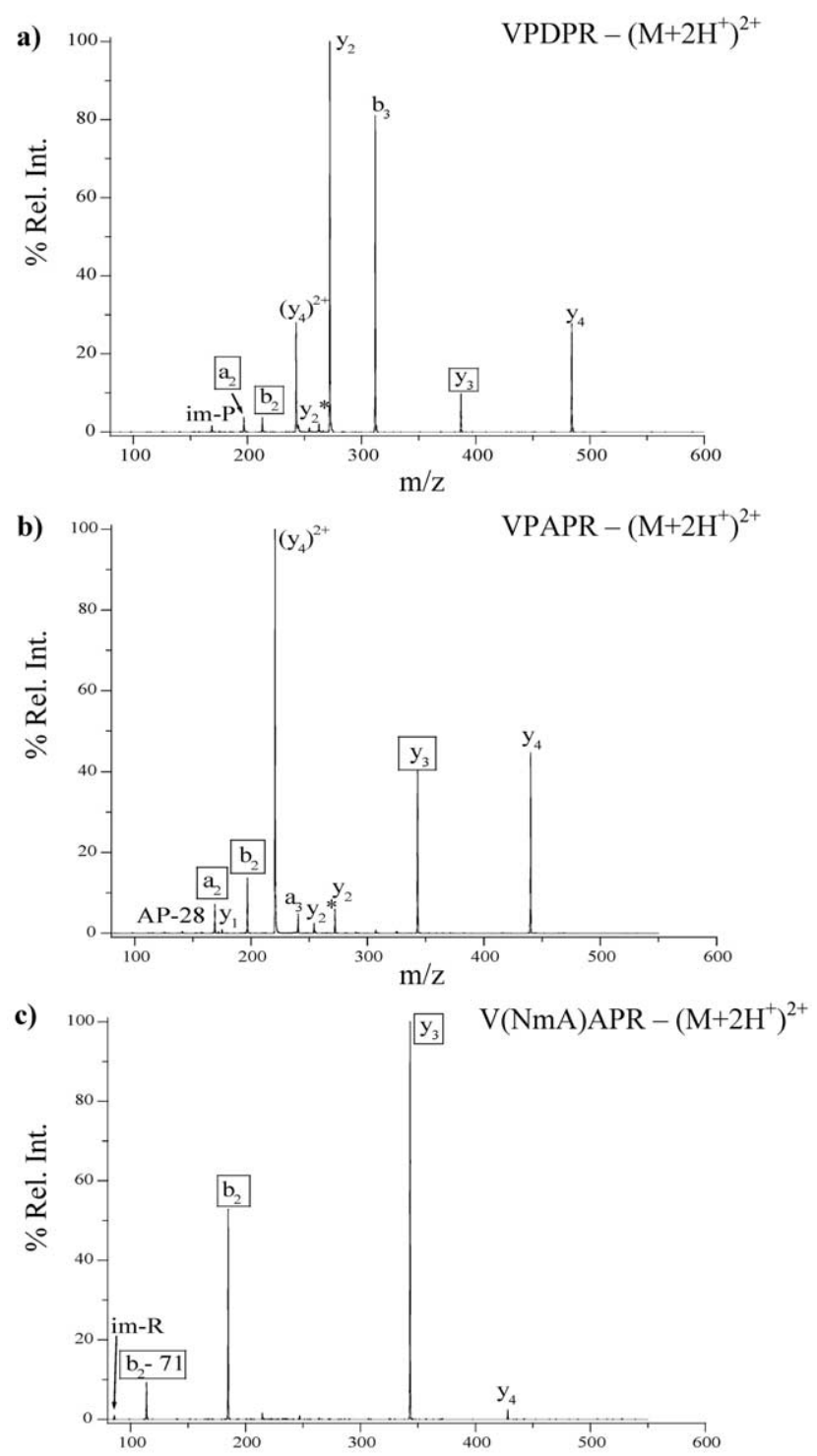

Figure 1. CID-LCQ spectra of doubly charged (a) VPDPR, (b) VPAPR, and (c) V(NmA)APR; im-X indicates an immonium for a given amino acid residue.

six-membered cyclic diketopiperazine rather than a five-membered oxazolone.

Previously established fragmentation trends for peptides containing proline fail to predict the formation of a predominant VP $b_{2}$ ion from collision induced dissociation of doubly charged VPDPR in a quadrupole ion trap. It has been speculated that an alternative $b_{2}$ ion structure, specifically a diketopiperazine rather than an oxazolone, may be formed. Farrugia et al. reported $\mathrm{MS}^{3}$ experiments that revealed the $b_{2}$ ion derived from either HG-Y or GH-Y (using single letter amino acid abbreviations) yielded identical spectra to the MS/MS spectrum of the protonated diketopiperazine of GH [46]. Furthermore, the side-chain protonated diketopiperazine of GH was thermodynamically favored over all other $b_{2}$ isomeric structures using $a b$ initio calculations. Additionally, Goolcharran and Borchardt reported the kinetic analysis of the conversion of FP- $p$-nitroaniline to FP-diketopiperazine in solution [47]. Among other solution phase properties that affected the rate of cyclization to a diketopiperazine structure (i.e., $\mathrm{pH}$, temperature, buffer concentration, and buffer species), the identity of the N-terminal amino acid residue played a major role in the kinetics. Specifically, the rate constant for degradation of Val-Pro- $p$-nitroaniline to ValPro-diketopiperazine was $116 \mathrm{M}^{-1} \mathrm{hr}^{-1}$, the second lowest rate constant of the residues investigated. The primary objective of the work presented here is to provide evidence for the stable gas-phase structure of a $\mathrm{b}_{2}$ ion containing proline as the C-terminal residue.

\section{Materials and Methods}

\section{Chemicals and Reagents}

All solvents used in peptide synthesis were purchased from Sigma-Aldrich (St. Louis, MO). NovaSyn amino acid residues and Wang resin beads were purchased from EMD Biosciences (San Diego, CA). Lyophilized VPDPR and acetic anhydride (to acetylate the N-terminus of the peptide sequence was purchased from Sigma (St. Louis). Cyclo-PV, a synthetic version of the diketopiperazine $\mathrm{VP} b_{2}$ ion (see inset for Figure $2 \mathrm{c}$ ), was purchased from BACHEM (Bubendorf, Switzerland).

\section{Peptide Synthesis and Chemical Modifications}

Solid-phase peptide synthesis was performed to synthesize VPAPR and V(NmA)APR (NmA is an abbreviation for N-methylalanine) using 9-fluorenylmethyloxycarbonyl (FMOC)-based chemistry. The C-terminal residue for both peptides was arginine and the synthesis was initiated from Wang resin beads to which PMC $(2,2,5,7,8-$ pentamethyl-chroman-6-sulphonyl)-protected arginine was coupled at a loading capacity of $0.54 \mathrm{mmol} \mathrm{g}^{-1}$. The resin was allowed to swell for $30 \mathrm{~min}$ in dimethyl formamide (DMF)/dichloromethane (DCM) (50:50) mixture before synthesis. Washing, deprotection with piperidine, and residue-coupling steps were completed for the Cterminal proline and alanine residues. The resin beads were then air-dried and divided equally to continue synthesis in two separate procedures, one involving the coupling of proline and finally valine and the second involving coupling of N-methyl alanine, followed by valine. After the synthesis was completed, a final deprotection step was performed (to form a free N-terminus) and cleavage of the synthesized peptide from the Wang resin beads was accomplished with a cleavage mixture of $95 \%$ trifluoroacetic acid with $2.5 \%$ (vol/vol) anisole added. The cleaved peptide (in solution) was separated from the solid resin beads by vacuum filtration, precipitated in $50 \mathrm{~mL}$ cold diethyl ether and furthered recovered through gravity filtration.

Acetylation of the free amine moiety at the N-terminus of each synthesized peptide was achieved by adding $5 \%$ (vol/vol) acetic anhydride in DMF to the peptide-resin 


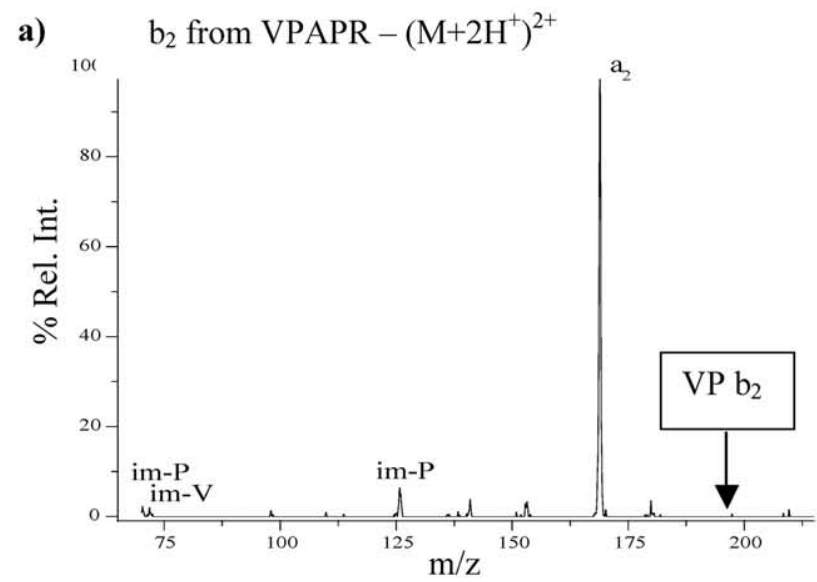

b) $\quad \mathrm{b}_{2}$ from $\mathrm{V}(\mathrm{NmA}) \mathrm{APR}-\left(\mathrm{M}+2 \mathrm{H}^{+}\right)^{2+}$
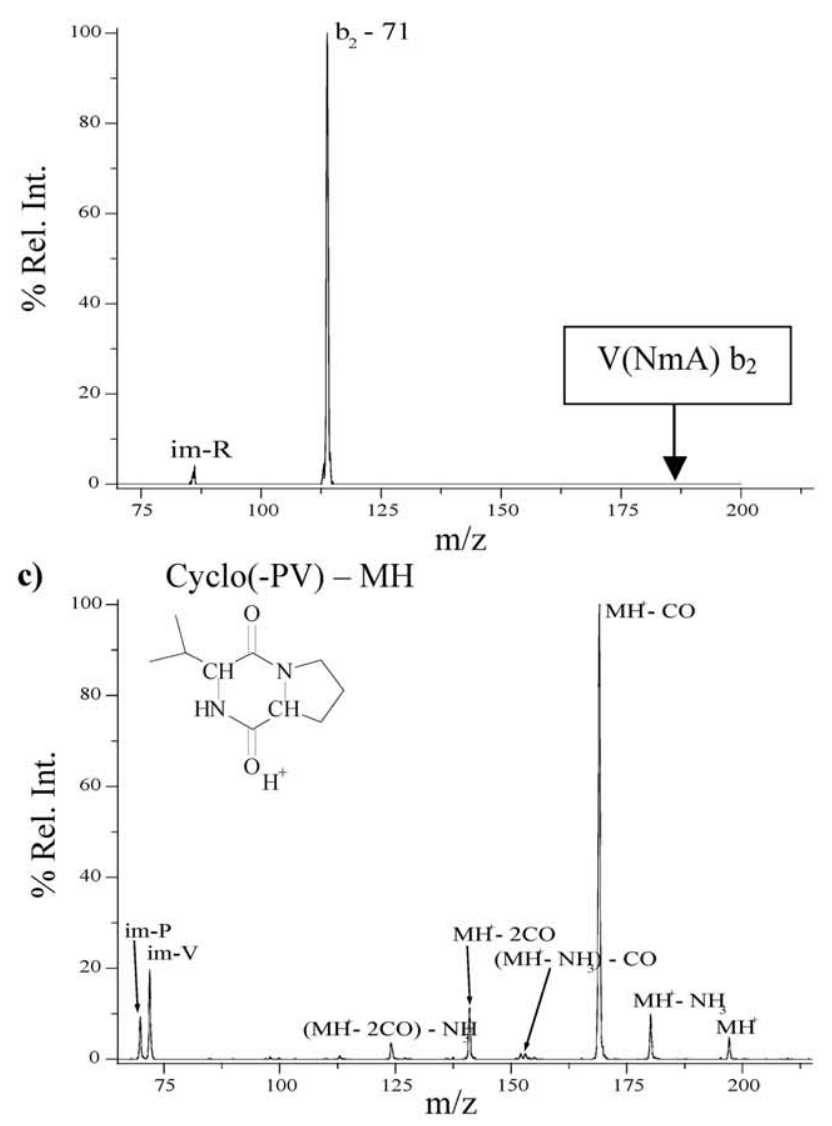

Figure 2. CID-LCQ MS/MS/MS spectra of $b_{2}$ ions originating from doubly charged (a) VPAPR ( $m / z$ 197.0), (b) V/NmA/APR ( $m / z$ 184.9), and (c) singly charged cyclo(-PV) ( $m / z$ 197.0); im-X indicates an immonium for a given amino acid residue.

beads following a final deprotection step (prior to the final cleavage from resin beads). Because the side chains of the residues were still protected by $t$-butyl groups, there was no acetylation to arginine residues.

\section{Instrumentation}

Low-energy collision induced dissociation experiments were performed in a quadrupole ion trap (Thermofinni- gan LCQ Classic, San Jose, CA) with parameters set for positive ion detection. Electrospray ionization (ESI) conditions included a needle voltage of $4.5 \mathrm{kV}$, nitrogen sheath gas flow rate of $\sim 8 \mu \mathrm{L} / \mathrm{min}$, a capillary voltage of $-10 \mathrm{~V}$ with a constant capillary temperature of $200{ }^{\circ} \mathrm{C}$. The lyophilized peptide was dissolved in $50 \%$ aqueous methanol with $1 \%$ acetic acid to yield a $10-30$ $\mu \mathrm{M}$ solution. The peptide solution was directly infused into the LCQ using a syringe pump at a rate of 2-3 $\mu \mathrm{L} / \mathrm{min}$. In the CID experiments, the incident ion energy was typically varied from 20 to $35 \%$ relative collision energy, as defined by Xcalibur software controlling the LCQ data acquisition.

High-resolution measurements were performed with an IonSpec 4.7 tesla Fourier transform ion cyclotron resonance (FT-ICR) mass spectrometer (Lake Forest, CA) equipped with an Analytica electrospray ionization (ESI) source (Branford, CT). The ions were introduced into the instrument by infusing $10-30 \mu \mathrm{M}$ solutions of the peptides in 50:50 methanol:water with $1 \%$ acetic acid using a stainless steel microelectrospray needle (0.004 in. i.d.) at a flow of $2-3 \mu \mathrm{L} / \mathrm{min}$. The source temperature was set at $200{ }^{\circ} \mathrm{C}$, and $3.8 \mathrm{kV}$ was applied to the ESI needle. The instrument has two regions of differential cryogenic pumping, referred to as the source and analyzer regions, with a typical analyzer base pressure of $7 \times 10^{-11}$ torr. The electrosprayed ions passed through a skimmer and were collected in an external RF-only hexapole, where they were allowed to accumulate for $400 \mathrm{~ms}$ before passing into the analyzer through a shutter. To perform fragmentation experiments, the desired ion was trapped inside the ICR cell by ejecting all other mass-to-charge values via a frequency sweep. Simultaneously applying a SORI pulse with a nitrogen gas pulse fragmented the isolated ion. Typical SORI conditions included a $2-3$ s pulse with $1-3 \mathrm{~V}$ amplitude at an offset of $1000 \mathrm{~Hz}$.

\section{Results and Discussion}

Collision induced dissociation of doubly charged VPDPR in a quadrupole ion trap (ThermoFinnigan LCQ Classic) produced a MS/MS spectrum showing cleavage at the second peptide bond, C-terminal to proline and N-terminal to aspartic acid (see Figure 1a). This fragmentation pattern contradicts expected residue specific behavior as determined by statistical analysis of fragmentation patterns for peptide MS/MS spectra for both proline and aspartic acid residues. While the predominant fragment ions present in the resulting CID-LCQ spectrum (see Figure 1a) are singly and doubly charged $y_{4}$ ions and $a b_{3} / y_{2}$ ion pair and follow expectations for proline-induced cleavages, there are other interesting peaks present in the spectrum that would not typically be predicted. In reality, the $b_{2}(+$ $\left.a_{2}\right) / y_{3}$ complementary ions that are present with significant relative intensities in the MS/MS spectrum would 
<smiles>CNC(C)C(=O)O</smiles>

\section{N-Methyl Alanine $(\mathrm{NmA})$}

Scheme 3. Chemical structures of neutral N-methyl alanine and proline.

not be expected based on fragmentation trends observed for larger peptides ( $>5$ residues) because these ions result from cleavage C-terminal to proline and cleavage $\mathrm{N}$-terminal to aspartic acid. However, the fragmentation pattern of doubly charged VPAPR (where aspartic acid is replaced by alanine) shows slightly enhanced formations of these same fragments, $b_{2}\left(+a_{2}\right) / y_{3}$ ions (see Figure $\left.1 b\right)$. It is concluded that the formation of these ions is not initiated by the presence of aspartic acid adjacent to proline in this peptide sequence. Formation of $b_{2}\left(+a_{2}\right) / y_{3}$ ions by fragmentation of VPAPR and $b_{2}\left(+a_{2}\right) / y_{3}$ ions by VPDPR are common to pentapeptides of the general sequence

XXXXR, yet the presence of proline as the C-terminal residue in the resulting $b_{2}$ ion is interesting. To understand the role proline plays in the formation of the $b_{2}(+$ $\left.\mathrm{a}_{2}\right) / \mathrm{y}_{3}$ complementary ions from CID of doubly charged VPAPR, the structural constraints that are unique to proline were omitted from the precursor ion by substituting $\mathrm{N}$-methyl alanine in the second sequence position (see Scheme 3) N-methyl alanine $(\mathrm{NmA})$ contains a tertiary nitrogen that mimics the basicity of the proline residue, yet the side-chain is not chemically bound to the peptide backbone, allowing a wider range of rotation about the single bonds in the residue and eases overall rotation of the peptide backbone.

MS/MS spectra obtained with CID in a quadrupole ion trap instrument are shown in Figure $1 \mathrm{~b}$ and $\mathrm{c}$ for doubly charged VPAPR and V(NmA)APR, respectively. The most striking differences between the fragmentation patterns of the two peptide ions include the absence of doubly charged $\mathrm{y}_{4}$ ion and the prevalence of $\mathrm{b}_{2} / \mathrm{y}_{3}$ complementary ions in the V(NmA)APR spectrum. The absence of $\left(\mathrm{y}_{4}\right)^{2+}$ ion and the decreased intensity of $\mathrm{y}_{4}$ ion in $\mathrm{V}(\mathrm{NmA}) \mathrm{APR}$ spectrum can be explained by the absence of the proline residue in the

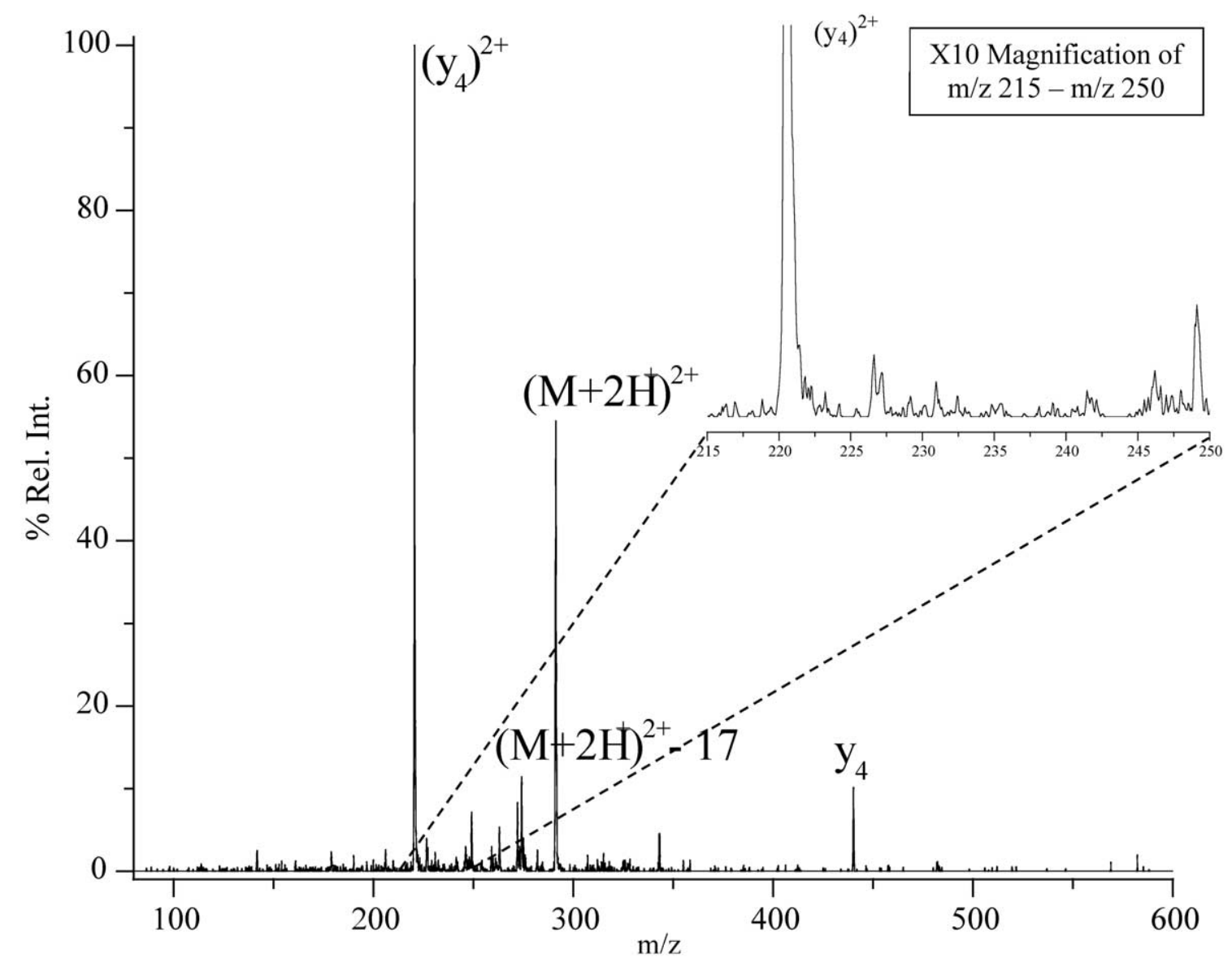

Figure 3. MS/MS of doubly charged N-acetylated VPAPR acquired by CID-LCQ. The absence of $\mathrm{m} / \mathrm{z}$ 239.9 indicates the free amino-terminus in VPAPR is involved in the formation of the $\mathrm{VP} \mathrm{b}_{2}$ fragment ion. 
sequence at the second position. Without cyclic proline in this position to direct cleavage to the $\mathrm{V}-\mathrm{P}$ peptide bond, fragment ions from this dissociation are no longer dominant. It should be noted that Vaisar and Urban have previously reported mono-N-methylated residues located internally in the peptide sequence may exhibit N-terminal preferential cleavage under low-energy collision induced dissociation conditions [41]. Despite this fact, cleavage occurring at the second peptide bond in doubly charged $\mathrm{V}(\mathrm{NmA}) \mathrm{APR}$ becomes the most prominent dissociation pathway, forming the $b_{2} / y_{3}$ complementary ions. Another interesting feature of the two spectra is the presence of different low mass ions. For VPAPR, the $b_{2}$ ion is paired with the formation of an $a_{2}$ ion (formal loss of $28 u$ from $b_{2}$ ) while the V(NmA)APR spectrum shows a peak with a $m / z$ value equivalent to a formal loss of $71 \mathrm{u}$ from the $\mathrm{b}_{2}$ ion. Through an $\mathrm{MS}^{3}$ experiment in which the $b_{2}$ ion formed from fragmentation of the corresponding doubly charged precursor ion is isolated and further fragmented, the structural characteristics of the $b_{2}$ ion may be determined. The fragmentation patterns of the respective $b_{2}$ ions in such an experiment [i.e., $\mathrm{VP} \mathrm{b}_{2}\left(m / z\right.$ 197.0) and $\mathrm{V}(\mathrm{NmA}) \mathrm{b}_{2}$ $(m / z$ 184.9)] were measured and are shown in Figure $2 \mathrm{a}$ and $b$, respectively. From the $\mathrm{MS}^{3}$ spectra, it can be concluded that the $b_{2}-71$ ion in the V(NmA)APR MS/MS spectrum and the $\mathrm{a}_{2}$ ion in the VPAPR MS/MS spectrum do originate from the respective $b_{2}$ ions rather than larger $b_{n}$ ions or the precursor ions.

The VP $b_{2}$ ion fragments to form not only the corresponding $\mathrm{a}_{2}$ ion, but also the immonium ions for proline $(m / z 70)$ and valine $(m / z 72)$. While it is not possible for the $\mathrm{V}(\mathrm{NmA}) \mathrm{b}_{2}$ ion to produce the immonium ion for proline (no proline in the sequence), there is also no formation of the valine immonium ion. It can be concluded from the vast differences in the resulting fragmentation patterns for each $b_{2}$ ion that the $\mathrm{V}(\mathrm{NmA})$ $b_{2}$ ion does not have the same gas-phase ion structure as the VP $b_{2}$ ion that is formed as a result of chargedirected dissociation of the initial protonated peptide ions [doubly charged V(NmA)APR and VPAPR, respectively].

In peptides where proline is located within 1 to 2 residues of the $\mathrm{N}$-terminus, involvement of the $\mathrm{N}$ terminal free amine in cleavage $\mathrm{C}$-terminal to proline may allow the formation of fragment ions that would not be predicted by the usual fragmentation patterns of proline-containing peptides (i.e., the observed $b_{2}(+$ $\left.\mathrm{a}_{2}\right) / \mathrm{y}_{3}$ ions from doubly charged VPDPR and VPAPR in Figure $1 \mathrm{a}$ and $\mathrm{b}$ ). The involvement of the N-terminal amine in formation of the $b_{2}$ ion would lead one to suspect the resulting gas-phase ion structure for the $b_{2}$ ion is a diketopiperazine. However, formation of the $a_{2}$ ion from dissociation of the corresponding $b_{2}$ ion, as observed in the fragmentation of VP $b_{2}$ ion, has been considered indicative of a protonated oxazolone structure that requires no participation of the free N-terminus for its formation [44]. It remains necessary to perform additional experiments to determine whether a
VP diketopiperazine can fragment to lose $\mathrm{CO}$ and form an $\mathrm{a}_{2}$ ion.

\section{Evidence for Diketopiperazine $V P b_{2}$}

Fragmentation of synthetic diketopiperazine $V P b_{2}$. One of the classic methods to determine proposed gas-phase ion structures is to fragment a synthetic compound that was chosen to have the same structure as the suspected structure. A comparison of the fragmentation patterns for the synthetic compound, assuming its structure does not change significantly upon ionization, and the ion in question will reveal similarities or differences in the two structures. As shown in Figure 2a and c, the fragmentation patterns for the VP $b_{2}$ ion formed initially by fragmentation of doubly charged VPAPR and for singly charged cyclo-PV are similar. The dominant fragmentation pathway involves the loss of carbon monoxide for both ions, resulting in $\mathrm{a}_{2}$ ion as the base peak in the $\mathrm{VP}_{2} \mathrm{MS}^{3}$ spectrum (Figure $2 \mathrm{a}$ ) and $\mathrm{MH}^{+}-\mathrm{CO}$ as the base peak in the cyclo-PV MS/MS spectrum (Figure 2c). Even more subtle features of the two spectra are identical: immonium ions are formed for both residues, proline and valine $(\mathrm{m} / \mathrm{z} 70$ and 72$)$ and peaks are present at $m / z$ values of 141,152 , and 180 , corresponding to sequential losses of water and ammonia (appropriate labels are provided in Figure 2c). It should be noted that there are minor differences between the two spectra. For example, singly charged, synthetic diketopiperazine VP produces a minor peak at $\mathrm{m} / \mathrm{z} 152$ that is absent from the $\mathrm{MS}^{3}$ spectrum for $\mathrm{VP} \mathrm{b}_{2}$ ion. Other differences between the pairs of spectra are the relative peak intensities for the immonium ions, loss of ammonia from the precursor ion $(\mathrm{m} / \mathrm{z} 180)$, and the loss equivalent to two CO molecules $(\mathrm{m} / \mathrm{z} 141)$.

The residual internal energy of the precursor ions resulting from ionization for cyclo(- PV) and from previous CID activation for the $\mathrm{VP} \mathrm{b}_{2}$ ion may contribute to these spectral differences. Additionally, there are likely to be differences in the precursor ion populations based on the presence of multiple protonation sites and the methods by which the precursor ions were formed (i.e., electrospray ionization from an acidified methanolic solution versus ion formation by collision induced dissociation of another ion). However, the high degree of similarity in the fragmentation patterns for a synthetic diketopiperazine VP ion and the VP $b_{2}$ ion provides further evidence the $\mathrm{VP} b_{2}$ ion does have $a$ gas-phase diketopiperazine structure.

N-terminal acetylation of VPAPR. As previously described, the N-terminal moiety must be a free amine to initiate formation of a diketopiperazine $b_{2}$ ion structure. By chemically modifying the free amino terminus with acetylation (resulting in $\mathrm{CH}_{3}(\mathrm{CO}) \mathrm{NH}$. . as the N-terminal functional group), the formation of a diketopiperazine $b_{2}$ ion structure can be prevented. The formation of a protonated oxazolone $b_{2}$ ion (originating from nucleophilic attack of the protonated carbonyl carbon by the 


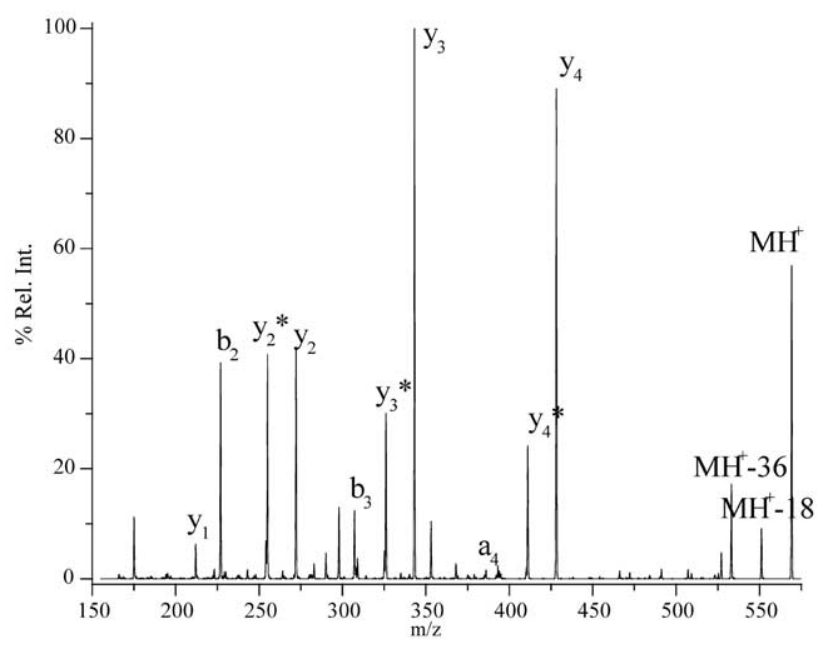

Figure 4. MS/MS of singly charged N-acetylated V/NmA/APR acquired by CID-LCQ. The presence of $m / z 227.4$ indicates the free amino-terminus in $\mathrm{V} / \mathrm{NmA} / \mathrm{APR}$ is not involved in the formation of the $\mathrm{V}(\mathrm{NmA}) \mathrm{b}_{2}$ fragment ion.

adjacent N-terminal carbonyl oxygen) would not be prevented by this chemical modification to the peptide sequence.

The MS/MS spectrum for doubly charged acetylated-VPAPR is given in Figure 3 . The $b_{2}$ ion is expected to occur at $m / z$ 239.9; the inset spectrum shows a magnified version of the MS/MS spectrum from $\mathrm{m} / \mathrm{z}$ 215 to 250 . No ion peaks at $\mathrm{m} / \mathrm{z} 239.9$ that are significantly higher than the spectral noise were detected. Absence of $a b_{2}$ ion when the $\mathrm{N}$-terminus is blocked is consistent with involvement of the free amine of the peptide $\mathrm{N}$-terminus in the formation of the $\mathrm{VP} \mathrm{b}_{2}$ ion. It can be concluded that the correct structure for this ion is a diketopiperazine or other ion formed by the involvement of the free $\mathrm{N}$-terminus.

\section{Evidence for Oxazolone $V(N m A) b_{2}$}

$N$-terminal acetylation of $V(N m A) A P R$. Chemical modification of the $\mathrm{N}$-terminus of the $(\mathrm{NmA})$-containing peptide sequence by acetylation should not affect the propensity of this precursor ion to fragment to form $a b_{2}$ ion if that ion is produced through the protonated oxazolone dissociation pathway. Recall that the formation of an oxazolone structure required attack of the adjacent $\mathrm{N}$-terminal carbonyl oxygen lone pair electrons on the electropositive carbonyl carbon at the site of protonation (see Scheme $\mathbf{1}$ ). If the $\mathrm{V}(\mathrm{NmA}) \mathrm{b}_{2}$ ion is an oxazolone, modification of the free amine on the N-terminus should have little or no affect on the formation of oxazolone $b_{n}$ ions and therefore, not prevent the formation of the $\mathrm{V}(\mathrm{NmA}) \mathrm{b}_{2}$ ion from the acetylated $\mathrm{V}(\mathrm{NmA}) \mathrm{APR}$ precursor ion.

Figure 4 shows the MS/MS spectrum from CID activation in the LCQ instrument for singly charged acetylated $\mathrm{V}(\mathrm{NmA}) \mathrm{APR}$. There is a strong $\mathrm{b}_{2}$ ion peak present at $\mathrm{m} / \mathrm{z} 227.4$, indicating that the acetylated
$\mathrm{N}$-terminal amine is not involved in the formation of this $b_{2}$ ion. Unfortunately, the low mass cutoff inherent in CID-LCQ spectra prevented the measurement of the corresponding $b_{2}-71$ ion. There is no way to determine if the N-terminal modification affected the formation of this ion from the CID-LCQ data. The identity of the $b_{2}-71$ ion is ambiguous with the level of mass accuracy that can be obtained with CID-LCQ data. Ideally, fragmentation of the doubly charged $\mathrm{V}(\mathrm{N}$ $\mathrm{mA}$ )APR precursor ion (rather than the singly charged ion) should have been used to evaluate the involvement of the N-terminal amine in the formation of the $\mathrm{V}(\mathrm{NmA}) b_{2}$ ion. Unfortunately, chemical alteration of the second most basic site on the pentapeptide (i.e., the N-terminal amine) significantly reduced the signal of the doubly charged ion to the point that only very poor quality MS/MS spectra (low signal-to-noise) could be acquired. Although a second proton is absent from the acetylated precursor ion, it is assumed that this proton is not critical to formation of the $\mathrm{V}(\mathrm{NmA}) b_{3}$ ion from the acetylated precursor ion because a larger $b_{2}$ ion is produced. Results from this experiment provide strong evidence that the $\mathrm{V}(\mathrm{NmA}) \mathrm{b}_{2}$ ion is an oxazolone.

Identification of $V(\mathrm{NmA}) b_{2}-71$ ion. There are two possible pathways by which the $\mathrm{V}(\mathrm{NmA}) b_{2}$ ion could formally lose a mass equivalent to $71 \mathrm{u}$, each of which originates from either the diketopiperazine or protonated oxazolone $\mathrm{V}(\mathrm{NmA}) \mathrm{b}_{2}$ ion structures. Two possible product ions with $m / z$ values equivalent to $b_{2}-71$ are shown in Scheme 4 and cannot be distinguished from one another at the level of mass accuracy available in CID-LCQ data $( \pm 0.5 \mathrm{u})$. Fortunately, the $\mathrm{b}_{2}-71$ ions possible from each $b_{2}$ structure have unique elemental compositions, providing a mass difference $(0.0364 \mathrm{u})$ that can be distinguished with high-resolution and mass accuracy measurements in a Fourier-transform mass spectrometer (FTMS). Figure 5 shows the MS/MS spectrum resulting from sustained off resonance irradiation-CID (SORI-CID) of singly charged V(NmA)APR measured with high mass accuracy and resolution. The poor signal intensity for doubly charged V(NmA)APR, again, prevented the use of the doubly charged precursor ion for SORI-CID measurements and it was assumed that the structural identity of the resulting $b_{2}-$ 71 ion was the same, regardless of the charge state of the (a)

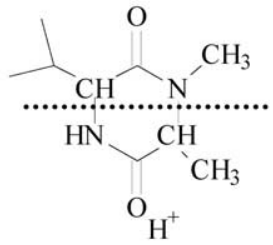

$\mathrm{C}_{6} \mathrm{H}_{12} \mathrm{NO}^{+}$

(114.0919u) (b)

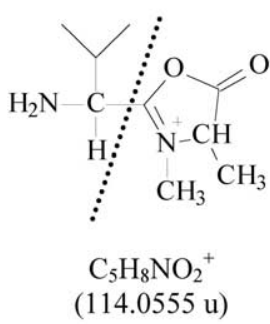

Scheme 4. Possible $b_{2}-71$ ions resulting from either (a) the diketopiperazine $b_{2}$ ion or (b) the oxazolone $b_{2}$ ion. 
precursor ion. The $b_{2}-71$ ion, produced by SORI-CID of singly charged $\mathrm{V}(\mathrm{NmA}) \mathrm{APR}$, has an accurate mass of $114.0553 \mathrm{u}$ when the MS/MS spectrum is internally calibrated using the immonium ion from arginine at $\mathrm{m} / \mathrm{z}$ 112.0900. This measured mass is closest to that of the $b_{2}$ - 71 ion arising from loss of a portion of the valine side-chain from the oxazolone $\mathrm{V}(\mathrm{NmA}) \mathrm{b}_{2}$ ion structure and is inconsistent with cross ring cleavage of the diketopiperazine structure (see inset for Figure 5), indirectly providing further evidence that the $\mathrm{V}(\mathrm{NmA}) \mathrm{b}_{2}$ ion is an oxazolone.

The $\mathrm{V}(\mathrm{NmA}) \mathrm{b}_{2}$ ion is an atypical oxazolone in that a corresponding $\mathrm{a}_{2}$ ion is not formed, but an ion equivalent to a formal loss of $71 \mathrm{u}$ from the $\mathrm{b}_{2}$ ion is formed instead. The $\mathrm{V}(\mathrm{NmA}) b_{2}$ ion is likely a methylated oxazolone, rather than the more common protonated oxazolone as shown in Scheme 1. The presence of the fixed charge (as a methyl group) and the absence of the proton on the ring nitrogen prevent intramolecular proton migration. The subsequent $b_{2}-71$ ion may be thought of as complementary to an $\mathrm{a}_{1}$ ion, which would otherwise form.

\section{Conclusions}

The loss of $\mathrm{CO}$, forming the corresponding $\mathrm{a}_{2}$ ion, has been considered a marker for a protonated oxazolone $b_{2}$ ion. Experimental evidence, including chemical modifi- cation of the N-terminus of the peptide and comparison of fragmentation patterns for the $b_{2}$ ion and a corresponding synthetic diketopiperazine, supports the suggestion that the $\mathrm{VP} \mathrm{b}_{2}$ ion is a diketopiperazine even though formation of the $\mathrm{a}_{2}$ ion is observed.

These results also directly support previous work by Eckart and coworkers in which a GP $b_{2}$ ion was determined to have a gas-phase structure different from the typical protonated oxazolone [48]. The overall stability of the $\mathrm{VP} \mathrm{b}_{2}$ diketopiperazine ion may encourage its formation if the transition-state structure is close to that of the product diketopiperazine structure [46]. $\mathrm{O}^{\prime} \mathrm{Hair}$ and coworkers suggested a side-chain protonation site on the histidine residue for a $\mathrm{GH} \mathrm{b}_{2}$ diketopiperazine ion stabilized the diketopiperazine structure. While VP $b_{2}$ has no such side-chain that would encourage charge retention (valine provides only a branched alkyl moiety), the overall stability of a 5, 6-membered bicyclic structure may encourage the formation of the diketopiperazine structure. Further stability calculations are needed to provide further evidence either for or against this speculation.

The larger impact of this work on automated sequencing relies on the refinement of the fragmentation trends observed for proline-containing peptide sequences in which enhanced cleavage $\mathrm{N}$-terminal to proline residues is expected. The cleavage C-terminal to

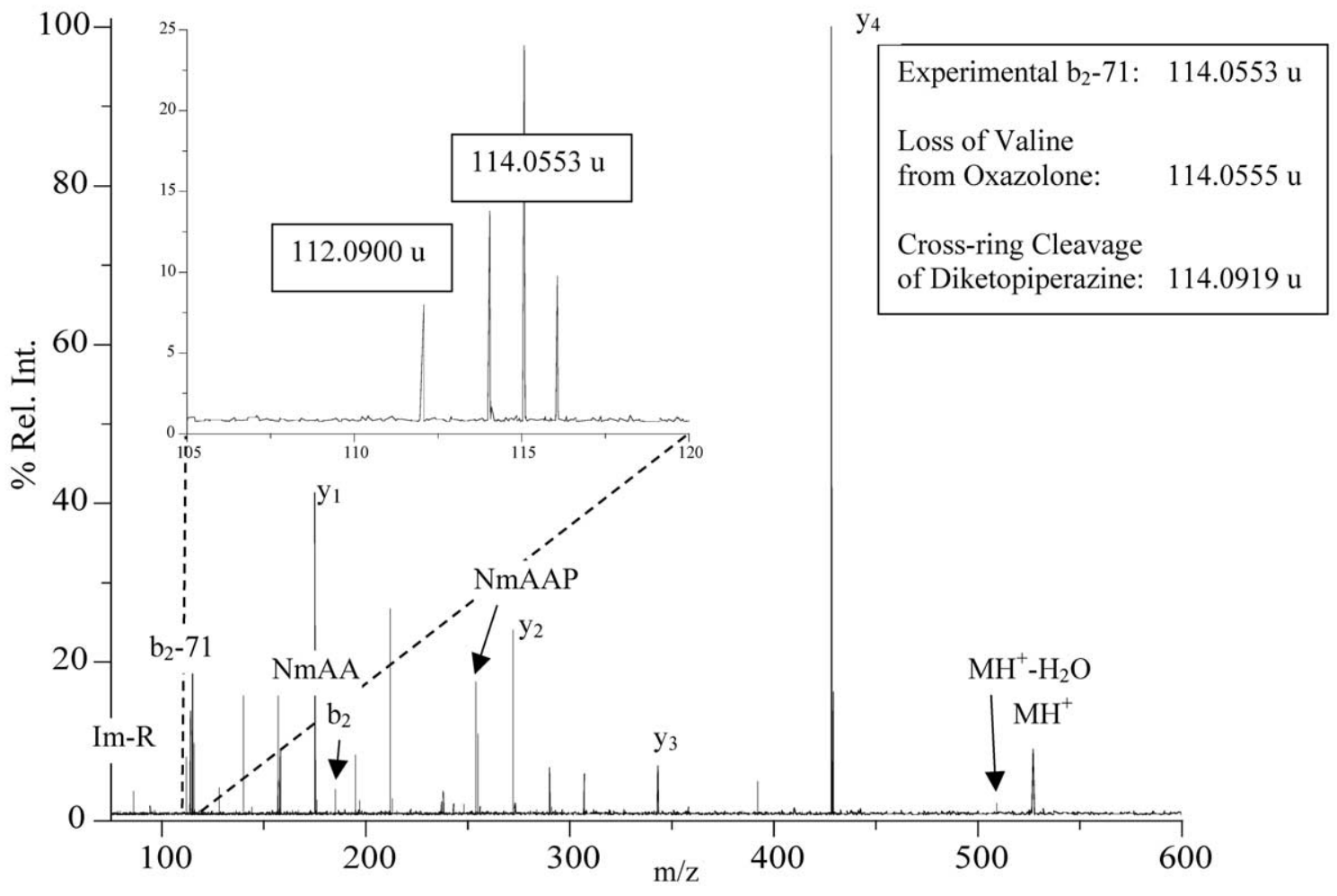

Figure 5. High-resolution measurements of $b_{2}-71$ fragment ion from SORI-CID/RE spectrum of singly charged VNmAAPR. Measured fragment ion $\mathrm{m} / \mathrm{z}$ value matches that of the loss of a portion of the valine residue from an oxazolone $b_{2}$ ion rather than the cross-ring cleavage of the isomeric diketopiperazine $b_{2}$ ion; im- $X$ indicates an immonium for a given amino acid residue. 
proline can and does occur in bioactive peptides as demonstrated by the presented research.

\section{Acknowledgments}

The authors thank the National Institutes of Health for funding of this research through grant GM R015387.

\section{References}

1. Breci, L.; Tabb, D. L.; Yates, J. R.; Wysocki, V. H. Cleavage N-terminal to proline: Analysis of a database of peptide tandem mass spectra. Anal. Chem. 2003, 75(9), 1963-1971.

2. Kapp, E. A.; Schutz, F.; Reid, G. E.; Eddes, J. S.; Moritz, R. L.; O'Hair, R. A. J.; Speed, T. P.; Simpson, R. J. Mining a tandem mass spectrometry database to determine the trends and global factors influencing peptide fragmentation. Anal. Chem. 2003, 75(22), 6251-6264.

3. Tabb, D. L.; Smith, L. L.; Breci, L. A.; Wysocki, V. H.; Lin, D.; Yates, J. R. Statistical characterization of ion trap tandem mass spectra from doubly charged tryptic peptides. Anal. Chem. 2003, 75(5), 1155-1163.

4. Huang, Y.; Triscari, J. M.; Wysocki, V. H.; Pasa-Tolic, L.; Anderson, G. A.; Lipton, M. S.; Smith, R. D. Dissociation behaviors of doublycharged tryptic peptides: Correlation of gas-phase cleavage abundance with Ramachadran plots. J. Am. Chem. Soc. 2004, 126(10), 3034-3035.

5. Aebersold, R.; Goodlett, D. R. Mass spectrometry in proteomics. Chem Rev. 2001, 101(2), 269-295.

6. Leibler, D. C. Introduction to proteomics: Tools for the new biology; Humana Press: Totowa, NJ, 2002; p 198.

7. Smith, R. D. Trends in mass spectrometry instrumentation for proteomics. Trends Biotechnol. 2002, 20(12), S3-S7.

8. Tyers, M.; Mann, M. From genomics to proteomics. Nature 2003, 422, 193-197.

9. Wu, C. C.; Yates, J. R. The application of mass spectrometry to membrane proteomics. Nat. Biotechnol. 2003, 21(3), 262-267.

10. Eng, J. K.; McCormack, A. L.; Yates, J. R. An approach to correlate tandem mass-spectral data of peptides with amino-acid-sequences in a protein database. J. Am. Soc. Mass Spectrom. 1994, 5(11), 976-989.

11. Perkins, D. N.; Pappin, D. J. C.; Creasy, D. M.; Cottrell, J. S. Probabilitybased protein identification by searching sequence databases using mass spectrometry data. Electrophoresis 1999, 20(18), 3551-3567.

12. Biemann, K. Contributions of mass spectrometry to peptide and protein structure. Biomed. Environ. Mass Spectrom. 1988, 16, 99.

13. Roepstorff, P.; Fohlman, J. Proposal for a common nomenclature for sequence ions in mass spectra for peptides. Biomed. Mass Spectrom. 1984, 11,601 .

14. Fenyo, D. Identifying the proteome: Software tools. Curr. Opin. Biotechnol. 2000, 11(4), 391-395.

15. Gatlin, C. L.; Eng J. K. Cross, S. T. Detter, J. C. Yates, J. R. Automated identification of amino acid sequence variations in proteins by HPLC/ microspray tandem mass spectrometry. Anal. Chem. 2000, 72(4), 757763.

16. Johnson, R. S.; Taylor, J. A. Searching sequence databases via de novo peptide sequencing by tandem mass spectrometry. Mol. Biotechnol. 2002, 22(3), 301-315.

17. Liska, A. J.; Shevchenko, A. Combining mass spectrometry with database interrogation strategies in proteomics. Trac-Trends Anal. Chem. 2003, 22(5), 291

18. Mann, M.; Hendrickson, R. C.; Pandey, A. Analysis of proteins and proteomes by mass spectrometry. Ann. Rev. Biochem. 2001, 70, 437-473.

19. Meyers, A.; Trauger, S.; Webb, W.; Reisdorph, N.; Wranik, C.; Peters, E.; Siuzdak, G. Protein identification and profiling with mass spectrometry. Spectrosc. Int. J. 2003, 17(1), 1-15.

20. Yates, J. R. Database searching using mass spectrometry data. Electrophoresis 1998, 19(6), 893-900.

21. Reid, G. E.; Simpson, R. J.; O’Hair, R. A. J. Int. J. Mass Spectrom. 1999, 190/191, 209

22. Huang, Y. Y.; Wysocki, V. H.; Tabb, D. L.; Yates, J. R. The influence of histidine on cleavage C-terminal to acidic residues in doubly protonated tryptic peptides. Int. J. Mass Spectrom. 2002, 219(1), 233-244.

23. Ambihapathy, K.; Yalcin, T.; Leung, H. W.; Harrison, A. J. Pathways to immonium ions in the fragmentation of protonated peptides. J. Mass Spectrom. 1997, 32, 209-215.

24. Arnott, D.; Kottmeir, D.; Yates, N.; Shabanowitz, J.; Hunt, D. F. Fragmentation of multiply protonated peptides under low energy conditions. Proceedings of the 42nd ASMS Conference on Mass Spectrometry; Chicago, IL, 1994.
25. Dongre, A. R.; Jones, J. L.; Somogyi, A.; Wysocki, V. H. Influence of peptide composition, gas-phase basicity, and chemical modification on fragmentation efficiency: Evidence for the mobile proton model. J. Am. Chem. Soc. 1996, 118(35), 8365-8374.

26. Gu, C. G.; Tsaprailis, G.; Breci, L.; Wysocki, V. H. Selective gas-phase cleavage at the peptide bond terminal to aspartic acid in fixed-charge derivatives of asp-containing peptides. Anal. Chem. 2000, 72(23), 58045813 .

27. Nair, H.; Wysocki, V. H. Are peptides without basic residues protonated primarily at the amino terminus? Int. J. Mass Spectrom. 1998, 174(1/3), 95-100.

28. Polce, M. J.; Ren, D.; Wesdemiotis, C. Special feature: Dissociation of the peptide bond in protonated peptides. J. Mass Spectrom. 2000, 35(12), 1391-1398.

29. Schlosser, A.; Lehmann, W. D. Five-membered ring formation in unimolecular reactions of peptides: A key structural element controlling low-energy collision-induced dissociation of peptides. J. Mass Spectrom. 2000, 35, 1382-1390.

30. Tsaprailis, G.; Somogyi, A.; Wysocki, V. H.; Zhong, W.; Futrell, J.; Summerfield, S. G.; Gaskell, S. J. Influence of secondary structure on fragmentation of protonated peptides. J. Am. Chem. Soc. 1999, 121, 5142-5154.

31. Wysocki, V. H.; Tsprailis, G.; Smith, L. L.; Breci, L. A. Mobile and localized protons: A framework for understanding peptide dissociation. J. Mass Spectrom. 2000, 35, 1399-1406.

32. Yague, J.; Paradela, A.; Ramos, M.; Ogueta, S.; Marina, A.; Barahona, F.; de Castro, J. A. L.; Vazquez, J. Peptide rearrangement during quadrupole ion trap fragmentation: Added complexity to MS/MS spectra. Anal. Chem. 2003, 75(6), 1524-1535.

33. Tsaprailis, G.; Somogyi, A.; Nikolaev, E. N.; Wysocki, V. H. Refining the model for selective cleavage at acidic residues in arginine-containing protonated peptides. Int. J. Mass Spectrom. 2000, 196, 467-479.

34. Wattenberg, A.; Organ, A. J.; Schneider, K.; Tyldesley, R.; Bordoli, R.; Bateman, R. H. Sequence dependent fragmentation of peptides generated by MALDI quadrupole time-of-flight (MALDI Q-TOF) mass spectrometry and its implications for protein identification. J. Am. Soc. Mass Spectrom. 2002, 13(7), 772-783.

35. Leymarie, N.; Berg, E. A.; McComb, M. E.; O'Connor, P. B.; Grogan, J.; Oppenheim, F. G.; Costello, C. E. Tandem mass spectrometry for structural characterization of proline-rich proteins: Application to salivary PRP-3. Anal. Chem. 2002, 74(16), 4124-4132.

36. Yalcin, T.; Csizamadia, I. G.; Peterson, M. R.; Harrison, A. J. The structure and fragmentation of bn $(\mathrm{n} \geq 3)$ ions in peptide spectra. J. Am. Soc. Mass Spectrom. 1996, 7, 233.

37. Yalcin, T.; Khouw, C.; Csizamadia, I. G.; Peterson, M. R.; Harrison, A. J. Why are $b$ ions stable species in peptide spectra? J. Am. Soc. Mass Spectrom. 1995, 6, 1164

38. Reid, G. E.; Simpson, R. J.; O'Hair, R. A. J. A mass spectrometric and ab initio study of the pathways for the dehydration of simple glycine and cysteine-containing peptide $[\mathrm{M}+\mathrm{H}]+$ ions. J. Am. Soc. Mass Spectrom. 1998, 9, 945.

39. Paisz, B.; Lendvay, G.; Vekey, K.; Suhai, S. Formation of b2+ ions from protonated peptides: An ab initio study. Rapid Commun. Mass Spectrom. 1999, 13, 525.

40. Nold, M. J.; Wesdemiotis, C.; Yalcin, T.; Harrison, A. J. Amide bond dissociation in protonated peptides. Structures of the N-terminal ionic and neutral fragments. Int. J. Mass Spectrom. Ion Processes 1997, 164, 137.

41. Vaisar, T.; Urban, J. Gas-phase fragmentation of protonated mono-Nmethylated peptides: Analogy with solution-phase acid-catalyzed hydrolysis. J. Mass Spectrom. 1998, 33(6), 505.

42. Vaisar, T.; Urban, J. Low-energy collisional induced dissociation of protonated peptides. Importance of an oxozolone formation for a peptide bond cleavage. Eur. J. Mass Spectrom. 1998, 4, 359.

43. Harrison, A. J.; Csizamadia, I. G.; Tang, T. H. Structure and fragmentation of b2 ions in peptide mass spectra. J. Am. Soc. Mass Spectrom. 2000, 11, 427-436.

44. Farrugia, J. M.; O'Hair, R. A. J.; Reid, G. E. Do all b2 ions have oxazolone structures? Multistage mass spectrometry and ab initio studies on protonated N-acyl amino acid methyl ester model systems. Int. J. Mass Spectrom. 2001, 210/211, 71-87.

45. Cordero, M. M.; Houser, J. J.; Wesdemiotis, C. Anal. Chem. 1993, 65, 1594.

46. Farrugia, J. M.; Taverner, T.; O'Hair, R. A. J, Side-chain involvement in the fragmentation reactions of the protonated methyl esters of histidine and its peptides. Int. J. Mass Spectrom. 2001, 209, 99-112.

47. Goolcharran, C.; Borchardt, R. T. Kinetics of diketopiperazine formation using model peptides. J. Pharm. Sci. 1998, 87(3), 283-288.

48. Eckart, K.; Holthausen, M. C.; Koch, W.; Spiess, J. Mass spectrometric and quantum mechanical analysis of gas-phase formation, structure, and decomposition of various b2 ions and their specifically deuterated analogs. J. Am. Soc. Mass Spectrom. 1998, 9, 1002-1011. 